\title{
Relieving emergency department crowding: Simulating the effects of improving patient flow over time
}

\author{
Eric Hamrock ${ }^{1}$, Kerrie Paige ${ }^{2}$, Jaret Hauge ${ }^{3}$, Jennifer Parks ${ }^{4}$, James Scheulen ${ }^{5}$, Scott Levin ${ }^{* 5,6}$ \\ ${ }^{1}$ Howard County General Hospital, Johns Hopkins Health System, Columbia, Maryland, USA \\ ${ }^{2}$ Healthcare Partners, General Electric, Seattle, Washington, USA \\ ${ }^{3}$ NovaSim, Seattle, Washington, USA \\ ${ }^{4}$ Casemix Information Management, Johns Hopkins Health System, Baltimore, Maryland, USA \\ ${ }^{5}$ Emergency Medicine, Johns Hopkins University School of Medicine, Baltimore, Maryland, USA \\ ${ }^{6}$ Operations Integration, Johns Hopkins Health System, Baltimore, Maryland, USA
}

Received: August 30, 2014

DOI: $10.5430 /$ jha.v4n1p43
Accepted: November 23, 2014 Online Published: December 9, 2014 URL: http://dx.doi.org/10.5430/jha.v4n1p43

\begin{abstract}
Background: Emergency Departments (ED) are challenged with excess demand for services and inadequate system capacity. Crowding at two independent EDs within a health system prompted an examination of the potential effects of improving patient throughput. The objective of this study was to determine the effects of reducing ED dwell time on temporal patterns of patient flow and demand for ED resources.

Methods: Separate discrete event simulation (DES) models were developed for the EDs of a 1,000-bed urban medical center and a 560-bed community medical center using patient flow information. These models characterized the effects of reducing patient dwell time on ED care area census (i.e., staffing needs), waiting room census, total length of stay (LOS) and waiting time. Dwell time was defined as the time interval from when a patient entered the main ED care area to when the patient exited the ED by discharge or hospital admission. Total LOS is defined as the entire time interval from ED from arrival to exit (including waiting time).

Results: DES results for each site demonstrate how natural patient arrivals and common hospital admission processes generate common temporal patterns of decreased crowding. Improving flow translates to most substantial reductions in waiting time and waiting room census during evening hours (17:00 to 22:00 hours). Significant effects on ED care area census and staffing demands are lagged, not occurring until overnight hours (2:00 to 8:00 hours). We reduced patient dwell time in 5\% increments within the urban ED (16.2 $\mathrm{min})$ and community $\mathrm{ED}(13.5 \mathrm{~min})$ from $5 \%$ to $15 \%$. For example, a $10 \%$ decrease in dwell time at the urban $\mathrm{ED}(32.4 \mathrm{~min})$ and community $\mathrm{ED}(27.0 \mathrm{~min})$ resulted in respective decreases in evening waiting room census by $49 \%$ (10.8 patients) and 26\% (3.5 patients) during evening hours and ED care area census by $16 \%$ (3.6 patients) and $11 \%$ (2.0 patients) overnight.

Conclusions: DES results suggest that increasing ED efficiency will most significantly decrease delays experienced by evening arrivals and provide opportunities to decrease care area census and reduce staff overnight.
\end{abstract}

Key Words: Emergency department crowding, Discrete event simulation, Patient flow

\footnotetext{
${ }^{*}$ Correspondence: Scott Levin; Email: slevin33@jhmi.edu; Address: Emergency Medicine, Johns Hopkins University, Davis Building Ste 3220 Baltimore, USA.
} 


\section{Introduction}

Crowding in emergency departments (EDs) hinders access, efficiency and the safety of care provided. ${ }^{[1]}$ Causes of ED crowding include both increases in volume and increased complexity of assessment and treatment provided in the ED. Although EDs and hospitals have little control over external forces that contribute to crowding, they do have internal opportunities to better manage resources and patient flow. EDs have the ability to alter physical space, staffing, and operational processes to more efficiently serve patients. Changes in any one of these areas may affect crowding, however the most successful interventions address all parts of the ED system and how they interact. ${ }^{[2]}$

The management of ED crowding is a major challenge for ED managers. Between 1993 and 2003, ED visits rose in the United States (US) by $26 \%$ from 90.3 million to 113.9 million. Yet, the number of hospital-based EDs decreased by 425 during the same period. ${ }^{[1]}$ Crowding is plaguing both urban and rural EDs alike. According to a national survey, $91 \%$ of EDs reported crowding as a problem with $40 \%$ indicating that it was a daily occurrence. ${ }^{[1]}$

ED volumes may be increasing in part because patients are having more difficulty accessing primary care and are turning to EDs to fill these unmet needs. ${ }^{[1,3]}$ Another potential cause of crowding may be linked to increased use of ED services by uninsured patients in need of healthcare. ${ }^{[4,5]}$ The Emergency Medical Treatment and Active Labor Act (EMTALA) ensures that all patients receive emergency care regardless of ability to pay. ${ }^{[6]}$ Recent expansion in healthcare insurance coverage brought on by the Affordable Care Act (ACA) is intended to address this problem; however, increased ED utilization for low acuity episodic illnesses are still present and have been observed for early adopters of health care reform. ${ }^{[7-10]}$ In addition to increased volume, demands on EDs are compounded by older and more complex patient populations requiring more time-consuming and resource intensive workups and treatments. ${ }^{[1]}$ Overall, crowding has been proven to decrease emergency care quality, patient safety, and satisfaction while increasing health care costs due to primary care needs being met in these suboptimal settings. ${ }^{[1,11,12]}$

Conceptually, ED operations may be broken down into 3 components: input, throughput, and output. ${ }^{[13]}$ Input is influenced by patient volume, complexity (i.e., resource needs) and natural arrival patterns much out of the EDs' control. Throughput comprises the time from when patients arrive to the ED, are triaged, evaluated, treated and ends when patients receive a final disposition. EDs have most direct control over the throughput component. Output includes time from final disposition to patients' discharge or admission as inpatients to the hospital. EDs may have some control over discharge processes. However, lack of inpatient beds creates boarding and bottlenecks in ED patient flow (i.e., throughput). Lack of inpatient beds has been consistently cited as a major source of ED crowding. ${ }^{[1]}$

EDs are charged with focusing on ways to most efficiently manage throughput. This includes improving operational processes that impact flow and best matching demand for resources (e.g., physicians, nurses, radiology, laboratory, etc.) to need for services. Matching demand to need ensures that specific resources do not create long waits prohibiting patient flow. Conversely, excessive and idle resources contribute to higher ED costs.

In the ED, efficiently managing resources, particularly staffing levels, is challenging due to temporal variation in demand for services. It is often difficult to examine these patterns in demand unless these data are readily available. Furthermore, understanding how patient flow may be affected by operational changes may be the ultimate goal but even more difficult to comprehend. Fortunately, tools from the field of systems engineering and operations research have been designed to examine flow systems and improve operational efficiency. The tools include queuing theory, discrete event simulation (DES), optimization, predictive modeling, and statistical process control and have been proven in other industries such as manufacturing, transportation, telecommunications, and finance. ${ }^{[1,14]}$

DES is a systems engineering tool used in this study to model patient flow at both EDs. DES allows for low risk assessment of operational changes prior to implementation and has previously been used in ED settings to examine patient flow and resource utilization. ${ }^{[14]}$ A study by Connelly and Bair (2004) focused on predicting patient throughput time. ${ }^{[15]}$ Other ED studies focused on analyzing nurse and physician staffing, patient length of stay (LOS), quality, and crowding. ${ }^{[16-18]}$ This study is distinct in examining the effects of improving patient throughput (i.e., reducing dwell time) on temporal patterns of crowding at both an urban and community ED.

This study aims to show the temporal effects of reducing ED dwell time at two separate EDs using DES. Dwell time was defined as the time interval from when a patient entered the main ED care area to when the patient exited the ED by discharge or hospital admission. Results of this study may guide ED administrators to identify opportunities for operational modifications due to changes in patient waiting time, waiting room census, and ED care area census (i.e., staffing needs). The study does not address what interventions should be implemented to reduce dwell time.

\section{Methods}

\subsection{Study design and setting}

This was a simulation study using retrospective patient flow data to simulate two diverse ED systems (i.e., urban and community). The urban academic ED is designated as a 
Level I trauma center and includes 25 main treatment beds, six psychiatric beds, seven urgent care (i.e., fast track areas), and a separate 14-bed observation unit. This ED sees an adult urban population of approximately 59,000 patients annually. The community ED is composed of 20 main treatment beds with eight urgent care areas and sees approximately 50,000 patients annually of both a considerably older adult population mixed with a pediatric patient population. This simulation study was conducted at both an urban academic and community ED with patient data being collected between September 2009 to January 2010 and January 2010 to December 2010, respectively. Each of these EDs was independently modeled using Simul $8_{T M}$ DES software.

\subsection{Measurements}

Simulation inputs included patient volumes, inter-arrival times, dwell time, disposition (i.e., inpatient admission/discharge) and bed capacity all stratified by service pathways (e.g., main care area, urgent care, psychology). These patient flow data were captured retrospectively via the electronic medical record and patient management (i.e., whiteboard) systems operating within each ED. These data were analyzed and transformed into probabilistic distributions which govern the flow (i.e., service time and routing) of patients through each simulation. These distributions assign routing such as patient service pathways and dispositions. Distributions for inter-arrival times and dwell time control timing of events within the simulation designed to mimic the temporal patterns and variability in flow experience in each ED modeled.

After each model reached final stages of development, verification and validation procedures were conducted. Input distributions were verified to match the patient flow data they were derived from. Critical outputs such as weekly patient census (i.e., occupancy) patterns and wait time distributions were examined to match each ED. Validated ED DES models were then altered by reducing dwell time in $5 \%$ increments from $5 \%$ to $15 \%$. This was done across all patients, discharged patients only, and admitted patients only (i.e., boarding time). Reductions of 5\% (5\% to $15 \%$ ) were chosen as a realistic, yet significant threshold for EDs to target. This scenario was designed to determine how increased throughput would temporally affect patient census and wait time over a 24-hour period.

It should be noted that verification and validation were conducted across all ED service pathways. However, results are focused on effects within the main ED care area at each institution due to the majority of patient volume and staffing resources focused in this area. In addition, these care areas are most generalizable to EDs across the United States.

Assumptions must be made when modeling the complexity of an ED using DES. This study assumed that patient volumes would remain in line with the base case (i.e., fixed) for both ED models regardless of changes in dwell time applied. The DES models for the urban and community ED differed in their assumption concerning left without being seen (LWBS) patients. LWBS were not taken into consideration in the urban ED DES model due to original study constraints. The community ED DES modeled LWBS by including these patients and representing their probability of leaving as a function of time. Longer patient waits resulted in linear increases in likelihood of LWBS and vice versa. The proportion of LWBS patients was an output included in the community ED model, but excluded for the urban ED model.

\section{Results}

Output from DES trials characterized how reducing dwell time in 5\% increments for all patients, admitted patients, and discharged patients resulted in reductions in crowding. The admission rate for the urban academic and community ED was $29 \%$ and $22 \%$, respectively. Table 1 displays how $5 \%$ decreases $(5 \%$ to $15 \%)$ in dwell time create sharp decreases in average wait time and total LOS. Total LOS is defined as the entire time interval from ED from arrival to exit (including waiting time). The relationship between dwell time and waiting time is evident, for example, a 5\% decrease in dwell time across all patients resulted in average waiting time decreases of 54\% (159.6 minutes) for the urban site and $10.9 \%$ (11.7 minutes) for the community site. Dwell times decreases were also applied to discharged patients or admitted patients in isolation as exhibited in Table 1. The urban ED observed a larger reduction in crowding metrics due to not directly accounting for LWBS patients.

Increased throughput translates to consistent patterns in ED care area and waiting room census over the course of a day. Figure 1 displays the temporal characteristics of reducing dwell times for all patients at both EDs. The waiting room is most significantly affected during evening hours (17:00 $22: 00)$ as seen by $5 \%$ decreases in dwell time resulting in $36 \%$ (7.8 patients) and 14\% (2.0 patients) decrease in average census at the urban and community hospitals, respectively. These hours coincide with peaks in waiting room census and average waiting time at both sites, and therefore, represent the greatest potential impact of improved throughput.

Within the model, the effects in the waiting room precede crowding effects in the care areas, similar to the natural sequence of patient movement through an ED. The $10 \%$ reductions in dwell times result in lagged decreases in ED care area census by $23 \%$ (4.7 patients) and $12 \%$ ( 2.10 patients) overnight (02:00 - 8:00 hours), at the urban and community hospitals respectively. This presents opportunities to lower staffing levels during this overnight time period, where labor rates are typically increased. ${ }^{[19]}$ 
Table 1: Effects of reducing ED dwell time

\begin{tabular}{|c|c|c|c|c|}
\hline \multirow[b]{2}{*}{ Model Description } & \multicolumn{2}{|c|}{ Urban ED } & \multicolumn{2}{|c|}{ Community ED } \\
\hline & $\begin{array}{l}\text { Total LOS (min) } \\
\text { Avg }\left(5^{\text {th }}-95^{\text {th }}\right) \\
\text { Percentile }\end{array}$ & $\begin{array}{l}\text { Waiting Time (min) } \\
\text { Avg }\left(5^{\text {th }}-95^{\text {th }}\right) \\
\text { Percentile }\end{array}$ & $\begin{array}{l}\text { Total LOS (min) } \\
\text { Avg }\left(5^{\text {th }}-95^{\text {th }}\right) \\
\text { Percentile }\end{array}$ & $\begin{array}{l}\text { Waiting Time (min) } \\
\text { Avg }\left(5^{\text {th }}-95^{\text {th }}\right) \\
\text { Percentile }\end{array}$ \\
\hline Baseline Dwell Time & $488.7(49.1-1296.6)$ & $297.5(19.5-897.3)$ & $368.8(105.8-862.1)$ & $107.5(0.0-594.6)$ \\
\hline 5\% Reduction in Dwell Time & - & - & - & - \\
\hline All Patients & $366.3(44.9-961.4)$ & $137.9(9.3-389.1)$ & 357.5 (104.5-838.7) & $95.8(0.0-550.0)$ \\
\hline Discharged Patients Only & $393.4(45.2-1059.3)$ & $179.5(11.2-534.0)$ & $363.4(104.9-873.0)$ & $101.8(0.0-576.3)$ \\
\hline Admitted Patients Only & $425.1(47.4-1056.1)$ & $198.5(16.1-497.3)$ & 364.2 (104.9-865.9) & $102.3(0.0-577.3)$ \\
\hline $10 \%$ Reduction in Dwell Time & - & - & - & - \\
\hline All Patients & $313.0(61.8-867.0)$ & $88.3(11.5-260.4)$ & 349.7 (104.1-806.9) & $80.5(0.0-496.7)$ \\
\hline Discharged Patients Only & $345.1(62.5-951.6)$ & $121.3(12.9-347.3)$ & $351.0(103.4-836.0)$ & $89.9(0.0-526.0)$ \\
\hline Admitted Patients Only & $378.8(67.8-1003.7)$ & $144.8(19.1-418.5)$ & $358.5(105.0-840.2)$ & $97.1(0.0-556.8)$ \\
\hline 15\% Reduction in Dwell Time & - & - & - & - \\
\hline All Patients & $282.5(73.4-814.6)$ & $63.5(12.7-169.8)$ & $325.8(101.5-757.1)$ & $70.1(0.0-452.0)$ \\
\hline Discharged Patients Only & 326.9 (76.3-938.9) & $111.0(18.8-363.2)$ & 346.9 (102.3-836.8) & $87.1(0.0-523.3)$ \\
\hline Admitted Patients Only & 350.2 (84.6-941.2) & $122.7(27.0-323.7)$ & 352.2 (105.1-816.7) & $90.8(0.0-529.9)$ \\
\hline
\end{tabular}
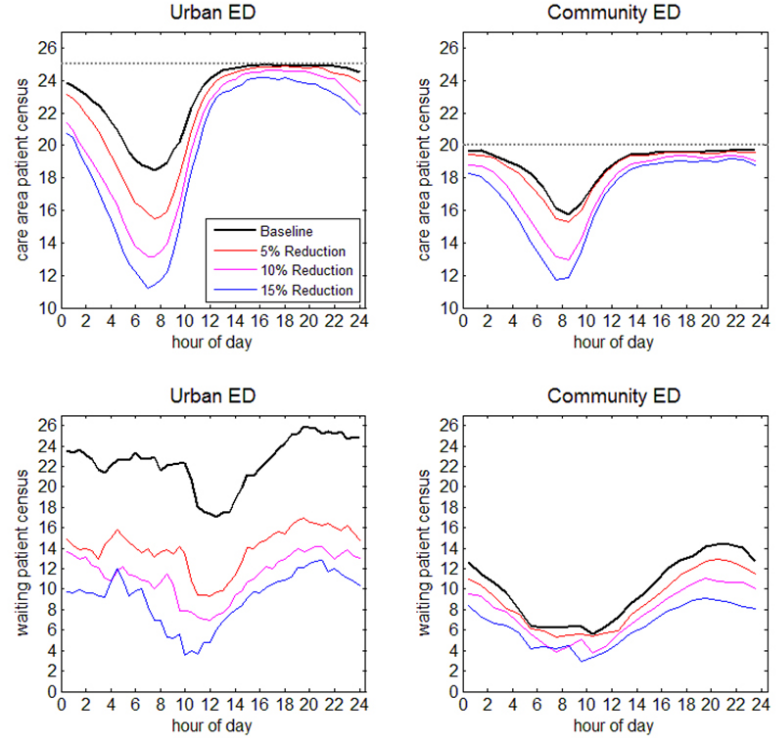

Figure 1: Effects of decreasing dwell time on crowding (care area and waiting room census) at the urban academic and community ED

\section{Discussion}

Increased utilization of ED services nationally has put stress on limited clinical resources at both community and academic medical centers. Efforts to improve ED efficiency and access commonly target increasing throughput (i.e., reducing dwell time). This usually involves altering operational processes, staffing and expanding bed capacity when possible. DES allows ED operational managers to see the magnitude and timing of reductions in crowding if improved efficiency is realized. This study characterized effects on all patients, discharged patients, and admitted patients separately. Management of discharged patients falls mostly under inter- nal control and may be addressed directly by the ED. Alternatively, admitted patients are boarded in the ED awaiting hospital access which often comprises a significant portion of their ED LOS. ${ }^{[20]}$ Although this waiting process is driven externally by hospital bed availability, it is useful to understand how reductions in boarding (i.e., decreases in LOS to admitted patients) will improve flow through the ED.

The sensitivity of crowding to reductions in dwell times for all patient groups is likely to be variable across EDs nationally. Characteristics including bed capacity, patient volume and complexity, staffing and information technology infrastructure will all mediate this relationship. Within DES, assumptions such as fixed demand (i.e., arrivals) and controlling for LWBS patients will also create variation in the simulated relationship between throughput (i.e., LOS) and crowding metrics. However, this study suggests that temporal patterns of crowding reductions resulting from decreased LOS will be fairly consistent across EDs in the United States. Patient census in care areas and waiting rooms exhibit similar patterns all related to timing of arrivals to the ED. Natural daily patient arrivals (i.e., unscheduled) patterns are somewhat predictable in both urban and community EDs nationally with peaks occurring in the evening between 4PM and 9PM and troughs occurring overnight between 3AM and 7AM. ${ }^{[21,22]}$ Arrival patterns for both study hospitals caring for very different populations were consistent with these national patterns. For this reason, we hypothesize that temporal patterns of crowding decreases associated with improving efficiency (i.e., reducing LOS) demonstrated in this study may be generalized.

\section{Limitations}

Limitations of this study included the DES at each site being originally designed for separate purposes, thus consid- 
erations such as LWBS and staffing considerations were not consistently accounted for across EDs. The study does not address what interventions may be implemented, but only the effects of successful interventions. Strengths of this study included the comparison of two EDs comprised of distinct patient populations and hospital types (urban academic medical center and community hospital) and the availability of temporal data to identify patient census and arrival patterns over a 24 hour period.

\section{Conclusion}

This study demonstrated that reductions in LOS had the greatest effects on ED patient census during non-peak hours. This effect may allow for reductions in overnight staffing levels thus optimizing scarce ED resources. Improving throughput will translate to most substantial crowding decreases in the waiting room during evening hours (17:00 to 22:00 hours), and these effects lagging in ED care areas overnight (2:00 to 8:00 hours). Urban and community EDs face significant challenges in managing excess demand for services and crowding. Improving efficiency by providing high quality care in less time (i.e., increasing throughput) at a lesser cost is a logical and widespread goal EDs strive for. This DES study at two independent EDs projected how incrementally reducing patient dwell times will translate to reductions in crowding in ED care areas and waiting rooms over a 24-hour period. Although magnitude of reductions in crowding will vary across EDs, patterns are likely general and consistent.

\section{References}

[1] Institute of Medicine. Hospital-Based Emergency Care: At the Breaking Point. Washington, DC: The National Academies Press; 2007. Available from: http://www.nap.edu/catalog.php?re cord_id $=11621$

[2] Institute of Medicine and National Academy of Engineering. Building a Better Delivery System: A New Engineering/Health Care Partnership. Washington, DC: The National Academies Press; 2005. Available from: http://www.nap.edu/catalog.php?record _id $=11378$

[3] Grumbach, K, Keane, D, Bindman, A. Primary Care and Public Emergency Department Overcrowding. American Journal of Public Health. 1993; 83 (3): 372-378. PMid: 8438975. http://dx.d oi .org/10.2105/AJPH. 83.3.372

[4] DeNavas-Walt C, Proctor B, Smith J, U.S. Census Bureau. Income, Poverty, and Health Insurance Coverage in the United States: 2012, U.S. Washington, DC: Government Printing Office; 2013. Available from: https://www. census.gov/hhes/www/hlthins/data/i ncpovhlth/2012/index.html

[5] Newton M, Carla K, Cunningham R, et al. Uninsured Adults Presenting to US Emergency Departments. Journal of the American Medical Association. 2008; 300(16): 1914-1924. PMid: 18940978. http://dx.doi.org/10.1001/jama.300.16.1914

[6] United States Department of Health and Human Services. Emergency Management Treatment and Labor Act (EMTALA) Overview. 198. Available from: http://www.cms.gov/Regulations-and-Guidance/Le gislation/EMTALA/index.html?redirect=/emtala/

[7] Effects on health insurance and the federal budget for the insurance coverage provisions in the Affordable Care Act. Congressional Budget Office; July, 2012 baseline. 2014. Available from: http://www.cbo.gov/publication/43900

[8] Pitts, S, Carrier, E, Kellerman, A. Where Americans Get Acute Care: Increasingly, It's Not At Their Doctor's Office. HealthAffairs. 2010; 29 (9): 1620-1629. PMid: 20820017. http://dx.doi.org $/ 10.1377 /$ hlthaff .2009 .1026

[9] Taubman SL, Allen HL, Wright BJ, et al. Medicaid Increases Emergency-Department Use: Evidence from Oregon's Health Insurance Experiment. Science. 2014; 343: 263-8. PMid: 24385603. http://dx.doi.org/10.1126/science. 1246183

[10] Smulowitz P, O'Malley J, Yang X, et al. Increased use of the emergency department after health care reform in Massachusetts. Annals of Emergency Medicine. 2014; 64(2): 107-115. PMid: 24656759. http://dx.doi.org/10.1093/heapol/czh031

[11] Hoot N, Aronsky D. Systematic review of emergency department crowding: causes, effects, solutions. Annals of Emergency
Medicine. 2008; 52(2); 126-136. PMid: 18433933. http://dx.d oi.org/10.1016/j. annemergmed.2008.03.014

[12] Berstein S, Aronsky D, Duseja R, et al. The Effect of Emergency Department Crowding on Clinically Oriented Outcomes. Academic Emergency Medicine. 2009; 16: 1-10. PMid: 19007346. http: //dx.doi.org/10.1111/j.1553-2712.2008.00295.x

[13] Asplin, B, Magid, D, Rhodes, K, et al. A Conceptual Model of Emergency Department Crowding. Annals of Emergency Medicine. 2003; 42: 173-180. PMid: 12883504. http://dx.doi.org/10. $1067 / \mathrm{mem} .2003 .302$

[14] Hamrock E, Paige K, Parks J, et al. Discrete event simulation for healthcare organizations: a tool for decision making. J Healthc Manag. 2013; 58(2): 110-124. Available from: http://www.nc bi.nlm.nih.gov/pubmed/23650696. PMid: 23650696.

[15] Connelly, L, Bair, A. Discrete Event Simulation of Emergency Department Activity: A Platform for System-level Operations Research. Academic Emergency Medicine. 2004; 11(11): 1177-1185. PMid: 15528582. http://dx.doi.org/10.1111/j.1553-271 2.2004.tb00702.x

[16] Raunak, M, Osterweil, L, Wise, A, et al. Simulating Patient Flow through an Emergency Department Using ProcessDriven Discrete Event Simulation. 2009 International Conference on Software Engineering (ICSE). Vancouver, Canada. Available from: http://ieeexplore.ieee.org/xpls/abs_all.jsp?a rnumber $=5069608 \&$ tag $=1$

[17] Hoot, N, Zhou, C, Jones, I, et al. Measuring and Forecasting Emergency Department Crowding in Real Time. Annals of Emergency Medicine. 2007; 49 (6): 747-755. PMid: 17391809. http://dx.d oi .org/10.1016/j. annemergmed.2007.01.017

[18] Hoot N, LeBlanc L, Jones I, et al. Forecasting Emergency Department Crowding: A Prospective, Real-Time Evaluation. J Am Med Inform Assoc. 2009; 16(3): 338-345. PMid: 19261948. http: //dx.doi.org/10.1197/jamia.M2772

[19] Stern and Associates. Emergency Physician Salary \& Benefit Survey: U.S National Results. 2009. Available from: www .aapsus.o $\mathrm{rg} /$ downloads $/ 2009 \% 20 \mathrm{National} \% 20$ Survey.pdf

[20] Levin, S, Dittus, R, Aronsky, D, et al. Optimizing Cardiology Capacity to Reduce Emergency Department Boarding: A Systems Engineering Approach. American Heart Journal. 2008; 156 (6): 12021209. PMid: 19033021. http://dx.doi.org/10.1016/j.ahj .2008 .07 .007

[21] Litvak, E, Long, M. Cost and Quality Under Managed Care: Irreconcilable Differences? The American Journal of Managed Care. 2000; 6 (3): 305-312. Available from: http://www.ajmc.com/publications/issue/2000/2000-0 3-vol6-n3/Mar00-661p305-312/. PMid: 10977431.

[22] McGaig, L, Burt, C. National Hospital Ambulatory Medical Care Survey. 2004. Emergency Department Summary. Available from: www.cdc.gov/nchs/data/ad/ad372.pdf 